\title{
Accelerated hyperfractionated intensity-modulated radiotherapy for recurrent/unresectable rectal cancer in patients with previous pelvic irradiation: results of a phase II study
}

Gang Cai, Ji Zhu, Weigang Hu and Zhen Zhang*

\begin{abstract}
Background: This study was conducted to investigate the local effects and toxicity of accelerated hyperfractionated intensity-modulated radiotherapy for recurrent/unresectable rectal cancer in patients with previous pelvic irradiation.

Methods: Twenty-two patients with recurrent/unresectable rectal cancer who previously received pelvic irradiation were enrolled in our single-center trial between January 2007 and August 2012. Reirradiation was scheduled for up to 39 Gy in 30 fractions using intensity-modulated radiotherapy plans. The dose was delivered via a hyperfractionation schedule of 1.3 Gy twice daily. Patient follow-up was performed by clinical examination, CT/MRI, or PET/CT every 3 months for the first 2 years and every 6 months thereafter. Tumor response was evaluated 1 month after reirradiation by CT/MRI based on the RECIST criteria. Adverse events were assessed using the National Cancer Institute (NCl) common toxicity criteria (version 3.0).

Results: The median time from the end of the initial radiation therapy to reirradiation was 30 months (range, 18-93 months). Overall local responses were observed in 9 patients (40.9\%). None of the patients achieved a complete response (CR), and 9 patients (40.9\%) had a partial response (PR). Thirteen patients failed to achieve a clinical response: 12 (54.5\%) presented with stable disease (SD) and 1 (4.5\%) with progressive disease (PD). Among all the patients who underwent reirradiation, partial or complete symptomatic relief was achieved in 6 patients (27.3\%) and 13 patients (59.1\%), respectively. Grade 4 acute toxicity and treatment-related deaths were not observed. The following grade 3 acute toxicities were observed: diarrhea (2 patients, 9.1\%), cystitis (1 patient, 4.5\%), dermatitis (1 patient, 4.5\%), and intestinal obstruction (1 patient, 4.5\%). Late toxicity was infrequent. Chronic severe diarrhea, small bowel obstruction, and dysuria were observed in 2 (9.1\%), 1 (4.5\%) and 2 (9.1\%) of the patients, respectively.

Conclusions: This study showed that accelerated hyperfractionated intensity-modulated radiotherapy significantly relieved local symptoms and led to a promising local response with an acceptable toxicity profile in patients with recurrent/unresectable rectal cancer and previous pelvic irradiation. Innovative treatment regimens should be evaluated in future studies to improve the clinical outcome while avoiding excessive toxicity in patients with recurrent rectal cancer and previous pelvic irradiation.
\end{abstract}

Keyword: Rectal cancer, Local recurrence, Reirradiation, Hyperfractionation, Intensity-modulated radiotherapy

* Correspondence: zhenzhang6@yahoo.com

Department of Radiation Oncology, Fudan University Shanghai Cancer Center, Shanghai 200032, China 


\section{Introduction}

Despite recent advances in pretreatment radiological evaluation, total mesorectal excision, radiotherapy, and chemotherapy, $5-10 \%$ of patients with primary rectal cancer develop locally recurrent rectal cancer (LRRC) [1-4]. LRRC can cause severe symptoms, including pelvic pain, bleeding, and bowel obstruction, which significantly impact the quality of life, and patient prognosis remains poor $[5,6]$. Salvage therapy for LRRC remains a challenge. Although radical surgery is the only approach with a curative intent, only approximately 20-30\% of patients with recurrent rectal cancer can undergo an R0 resection [7]. Most patients cannot undergo curative surgery because of locally unresectable disease, lack of medical fitness, an unwillingness to accept the high postoperative morbidity and mortality rates [7], or the presence of unresectable extrapelvic metastases.

Radiation is an effective approach for relieving symptoms and improving local control in patients with recurrent rectal cancer. However, some patients with recurrent rectal cancer have previously received radiation, which increases the complexity and difficulty of the management. Reirradiation is often not recommended because of the extremely high incidence of complications in normal tissues. Although data regarding reirradiation outcomes are scarce, several recent observations and trials have demonstrated the safety and efficacy of reirradiation in patients with recurrent rectal cancer who previously underwent irradiation [8-13]. To reduce the potential risk of late toxicity in normal tissues related to previous radiation, hyperfractionated radiation may be a good option. In addition, NCCN guidelines recommend that intensitymodulated radiotherapy (IMRT) be used in the context of reirradiating patients with recurrent disease [14]. However, to the best of our knowledge, no prospective studies have evaluated the efficacy and safety of accelerated hyperfractionated IMRT in patients with recurrent rectal cancer and previous pelvic irradiation. Based on these considerations, we performed a phase II study to investigate the local effects and safety of accelerated hyperfractionated IMRT in patients with recurrent rectal cancer who previously received pelvic irradiation.

\section{Materials and methods \\ Patients}

Twenty-two patients with recurrent/unresectable rectal cancer and previous pelvic irradiation were enrolled between January 2007 and August 2012 in our single-center trial. This prospective study was approved by our institutional review board (Fudan University Shanghai Cancer Center), and all the patients signed informed consent forms.

To be eligible, patients had to present with histologically confirmed primary rectal adenocarcinoma. Each patient was discussed, evaluated, and determined to have unresectable cancer by our multidisciplinary team before reirradiation, and a pelvic recurrence was diagnosed by histological confirmation or based on the typical appearance on PET/ computed tomography (CT), CT, or MRI. All the patients had received previous pelvic irradiation. There were measurable lesions in the fields of radiotherapy. The other inclusion criteria were as follows: age between 18 and 75 years; Eastern Cooperative Oncology Group performance score $\leq 2$; adequate hematological and liver function; leucocytes $>4.0 \times 10^{9} / \mathrm{L}$; platelets $>100 \times 10^{9} / \mathrm{L}$; bilirubin $<1.5 \times$ the upper limit of normal (ULN); aspartate aminotransferase/alanine aminotransferase $\leq 2.5 \times$ ULN; and serum creatinine $<1.25 \times$ ULN.

The exclusion criteria were as follows: any other malignancy; significant coronary or cardiac conditions; serious uncontrolled infection; a psychiatric disorder; and pregnancy or lack of contraceptive use in women with childbearing potential.

\section{Pretreatment evaluation}

The pretreatment workup was performed within the two weeks prior to initiating the reirradiation and included a complete history, physical examination, digital rectal examination, colonoscopy (if possible), tumor biopsy (if possible), chest CT, abdominal CT, pelvic CT or MRI or PET/CT, and complete laboratory tests.

\section{Radiotherapy}

Radiotherapy was delivered with a linear accelerator using 6-MV photons. Each patient had a planning CT scan in the treatment position (prone position using a belly board or supine position). IMRT planning was performed for all the patients based on the planning CT. Target definition followed the recommendations of ICRU report No. 83 [15]. The target volumes and the nearby at-risk organs were delineated on the Pinnacle 8.0 planning system. The gross tumor volume (GTV) was determined based on a combination of the findings from the physical exam, CT, MRI, and/or PET/CT. The planning target volume (PTV) was generated with a $2-3 \mathrm{~cm}$ margin around the GTV to avoid as much of the bladder and small bowel as possible. The treatment plans were optimized such that more than 98\% of the PTV was completely encompassed by the $93 \%$ isodose line while maintaining a minimum dose greater than $90 \%$ and a maximum dose less than $110 \%$. The small bowel, bladder, and femoral heads were defined as at-risk organs. The tolerances of normal tissues were defined as follows: 1) Small bowel: No more than 180 cc above $20 \mathrm{~Gy}$, no more than $65 \mathrm{cc}$ above $30 \mathrm{~Gy}$, and maximum dose less than 40 Gy; 2) Femoral heads: No more than $40 \%$ volume above 25 Gy, no more than $25 \%$ volume above $30 \mathrm{~Gy}$, and maximum dose less than $40 \mathrm{~Gy}$; and 3) Bladder: No more than $40 \%$ volume above 25 Gy, no more 
than $15 \%$ volume above $30 \mathrm{~Gy}$, and maximum dose less than 40 Gy. Inverse planning with five coplanar IMRT fields was constructed. The dose was delivered twice daily, 5 days per week, with a hyperfractionation schedule of $1.3 \mathrm{~Gy}$ and an interval of at least $6 \mathrm{~h}$ between fractions. Thirty fractions to 39 Gy were prescribed.

\section{Treatment after reirradiation}

After reirradiation, subsequent treatment was recommended as necessary but was not included in the study protocol. Subsequent treatment (chemotherapy or surgery) was individualized with no specific recommendations.

\section{Study design and data evaluation}

The primary endpoints of this study were local response and symptom relief after accelerated hyperfractionated IMRT for recurrent rectal cancer in patients with previous pelvic irradiation. Secondary endpoints included toxicity and follow-up.

The patients were routinely followed with clinical examinations, CT/MRI, or PET/CT every 3 months for the first 2 years and every 6 months thereafter. Tumor responses were evaluated 1 month after reirradiation by CT/MRI according to the RECIST criteria. Adverse events were assessed using the National Cancer Institute (NCI) common toxicity criteria (version 3.0).

The different parameters for each individual patient were entered into a database and were analyzed using SPSS 17.0 statistical software. Local progression-free survival rates and overall survival rates were determined using the Kaplan-Meier method.

\section{Results}

Patient characteristics

Twenty-two patients with recurrent rectal cancer who previously received pelvic irradiation were enrolled between January 2007 and August 2012 in our single-center trial. The study population had a median age of 53 years (range, 40-68 years). Nine participants were men (40.9\%), and 13 (59.1\%) were women.

Table 1 summarizes the patient characteristics at primary diagnosis and their prior treatment. The initial staging was as follows: 3 stage I patients, 2 stage II patients, and 17 stage III patients. A total of 11 patients $(50.0 \%)$ underwent low anterior resection, 10 (45.5\%) underwent abdominoperineal resection, and 1 (4.5\%) underwent local excision. In addition, 17 patients received postoperative chemotherapy consisting of capecitabine or 5-FU plus oxaliplatin. Previous irradiation consisted of preoperative irradiation in 3 patients, postoperative irradiation in 11 patients, and irradiation at first recurrence in 8 patients. The previous radiation doses to the pelvis ranged from 36 to $62 \mathrm{~Gy}$, with a median of $48.6 \mathrm{~Gy}$.
Table 1 Patient characteristics at primary diagnosis and previous treatment $(\mathbf{n}=\mathbf{2 2})$

\begin{tabular}{|c|c|c|}
\hline Characteristics & No. & $\%$ \\
\hline \multicolumn{3}{|l|}{ Gender } \\
\hline Male & 9 & 40.9 \\
\hline Female & 13 & 59.1 \\
\hline \multicolumn{3}{|l|}{ T stage at primary diagnosis } \\
\hline $\mathrm{T} 1$ & 4 & 18.2 \\
\hline $\mathrm{T} 2$ & 1 & 4.5 \\
\hline $\mathrm{T} 3$ & 12 & 54.5 \\
\hline T4 & 5 & 22.7 \\
\hline \multicolumn{3}{|l|}{ Primary N stage } \\
\hline No & 5 & 22.7 \\
\hline N1 & 9 & 40.9 \\
\hline $\mathrm{N} 2$ & 8 & 36.4 \\
\hline \multicolumn{3}{|l|}{ Primary stage } \\
\hline 1 & 3 & 13.6 \\
\hline$\|$ & 2 & 9.1 \\
\hline III & 17 & 77.3 \\
\hline \multicolumn{3}{|l|}{ Initial surgery } \\
\hline Low anterior resection & 11 & 50.0 \\
\hline Abdominoperineal resection & 10 & 45.5 \\
\hline Local excision & 1 & 4.5 \\
\hline \multicolumn{3}{|l|}{ Previous radiation } \\
\hline Preoperative & 3 & 13.6 \\
\hline Postoperative & 11 & 50.0 \\
\hline Recurrent & 8 & 36.4 \\
\hline \multicolumn{3}{|l|}{ Previous radiation dose } \\
\hline Median (Gy) & 48.6 & - \\
\hline Range (Gy) & $36-62$ & - \\
\hline
\end{tabular}

The patient characteristics at reirradiation are presented in Table 2 . The interval between the initial surgery and the first pelvic recurrence ranged from 13 to 100 months, with a median of 29 months. The median time from the end of the initial radiation to reirradiation was 30 months (range, 18-93 months). Four patients with recurrent rectal cancer had simultaneous extrapelvic metastases at the time of reirradiation, whereas 18 patients did not present with extrapelvic metastasis. Thirteen patients underwent reirradiation at the first recurrence, and 9 patients underwent reirradiation at the second recurrence. There were a total of twenty-five recurrent sites among all the patients: 20 patients had a single site, and 2 patients had multiple sites. With respect to location, 5 sites were in the perirectal region, 7 in the presacral region, 7 in the internal iliac nodal region, 5 in the perineum, and 1 in the external iliac nodal region. After reirradiation, 18 patients received 5-FU 
Table 2 Patient characteristics at reirradiation $(n=22)$

\begin{tabular}{|c|c|c|}
\hline Characteristics & No. & $\%$ \\
\hline \multicolumn{3}{|l|}{ Age } \\
\hline Median (y) & 53 & - \\
\hline Range (y) & $40-68$ & - \\
\hline \multicolumn{3}{|l|}{ Recurrent sites } \\
\hline Single & 20 & 90.9 \\
\hline Multiple & 2 & 9.1 \\
\hline \multicolumn{3}{|l|}{ Recurrence location ( $n=25^{*}$ ) } \\
\hline Perirectal region & 5 & 20.0 \\
\hline Presacral region & 7 & 28.0 \\
\hline Internal iliac nodal region & 7 & 28.0 \\
\hline Perineum & 5 & 20.0 \\
\hline External iliac nodal region & 1 & 4.0 \\
\hline \multicolumn{3}{|l|}{ Frequency of recurrence } \\
\hline First recurrence & 13 & 59.1 \\
\hline Second recurrence & 9 & 40.9 \\
\hline \multicolumn{3}{|l|}{ Reirradiation interval } \\
\hline Median (months) & 30 & - \\
\hline Range (months) & $18-93$ & - \\
\hline$\leq 2$ year & 7 & 31.8 \\
\hline$>2$ year & 15 & 68.2 \\
\hline \multicolumn{3}{|l|}{ Size of largest recurrence } \\
\hline$\leq 3 \mathrm{~cm}$ & 5 & 22.7 \\
\hline$>3 \mathrm{~cm}$ & 17 & 77.3 \\
\hline \multicolumn{3}{|l|}{ CEA level } \\
\hline Normal (<5 ng/mL) & 4 & 18.2 \\
\hline Increased ( $\geq 5$ ng/mL) & 18 & 81.8 \\
\hline \multicolumn{3}{|l|}{ Extra pelvic metastases } \\
\hline No & 18 & 81.8 \\
\hline Yes & 4 & 18.2 \\
\hline \multicolumn{3}{|c|}{ Chemotherapy after reirradiation } \\
\hline No & $4^{* *}$ & 18.2 \\
\hline Yes & 18 & 81.8 \\
\hline
\end{tabular}

${ }^{*}$ Multiple locoregional recurrences present in 2 patients (the perirectal region and the perineum in one patient; the presacral region, internal iliac nodal region, and perineum in another patient).

**Subsequent chemotherapy was recommended for all patients, but 4 patients did not undergo chemotherapy due to intestinal obstruction (2 patients), progressive disease during reirradiation (1), and refusal (1).

based chemotherapy, and none of these patients underwent a subsequent surgical resection.

\section{Local response and clinical symptom relief}

All the patients with recurrent rectal cancer who previously underwent pelvic irradiation were evaluated for local responses 1 month after reirradiation using CT/MRI. In total, 9 patients (40.9\%) achieved a partial response (PR); there was no complete response. Thirteen patients failed to achieve a clinical response, with 12 (54.5\%) presenting with stable disease (SD), and 1 (4.5\%) presenting with progressive disease (PD) (Table 3).

All the patients had severe clinical symptoms before reirradiation, including pain (17 patients), bleeding (1 patient), mass effect ( 1 patient), pain and bleeding ( 2 patients), and bleeding and mass effect (1 patient). Complete or partial clinical symptom relief after reirradiation was achieved in 6 patients (27.3\%) and 13 patients (59.1\%), respectively. Clinical symptom palliation was achieved for a median duration of 10 months (range, 3-20 months) (Table 3).

\section{Toxicity and dose intensity}

Table 4 shows the incidence of acute toxicity during reirradiation. Grade 4 toxicity and treatment-related deaths were not observed. Diarrhea was the most common side effect. Grade 3 toxicities included diarrhea in 2 patients (9.1\%), cystitis in 1 patient (4.5\%), radiation dermatitis in 1 patient (4.5\%), and an intestinal obstruction in 1 patient (4.5\%).

A total of 20 patients $(90.9 \%)$ received the planned dose (39 Gy) of accelerated hyperfractionated reirradiation as scheduled (mean relative dose intensity, 95.8\%). The reirradiation was terminated for 2 patients: one developed a grade 3 intestinal obstruction, and the other experienced progressive disease. The one patient with a grade 3 intestinal obstruction achieved symptom resolution after adopting conservative treatment. Six patients (27.3\%) required a break in the reirradiation course, with a median delay of 2 days (range, 1-6 days), due to acute toxicity.

The incidence of late toxicity was infrequent. Only 2 patients developed severe chronic diarrhea. One patient developed a small bowel obstruction and was hospitalized for occasional tube decompression. Two patients developed dysuria and required percutaneous nephrostomy drainage. None of the patients developed skin ulceration.

\section{Table 3 Local response rates and clinical symptom relief}

\begin{tabular}{lll}
\hline Characteristics & No. & $\%$ \\
\hline Local response & 0 & 0 \\
Complete response (CR) & 9 & 40.9 \\
Partial response (PR) & 12 & 54.5 \\
Stable disease (SD) & 1 & 4.5 \\
Progressive disease (PD) & & \\
Symptom relief* & 6 & 27.3 \\
Complete relief & 13 & 59.1 \\
Partial relief & 3 & 13.6 \\
No relief &
\end{tabular}

*All of the patients had severe clinical symptoms before reirradiation, including pain (17 patients), bleeding (1 patient), mass effect (1 patient), pain and bleeding ( 2 patients), and bleeding and mass effect ( 1 patient). 
Table 4 Acute toxicity

\begin{tabular}{lllll}
\hline Toxicity & $\begin{array}{l}\text { Grade 1 } \\
\text { no. (\%) }\end{array}$ & $\begin{array}{l}\text { Grade 2 } \\
\text { no. (\%) }\end{array}$ & $\begin{array}{l}\text { Grade 3 } \\
\text { no. (\%) }\end{array}$ & $\begin{array}{l}\text { Grade 4 } \\
\text { no. (\%) }\end{array}$ \\
\hline Diarrhea & $3(13.6)$ & $4(18.2)$ & $2(9.1)$ & $0(0)$ \\
Cystitis & $2(9.1)$ & $4(18.2)$ & $1(4.5)$ & $0(0)$ \\
$\begin{array}{l}\text { Radiation } \\
\text { dermatitis }\end{array}$ & $0(0)$ & $1(4.5)$ & $1(4.5)$ & $0(0)$ \\
$\begin{array}{l}\text { Intestinal } \\
\text { obstruction }\end{array}$ & $0(0)$ & $0(0)$ & $1(4.5)$ & $0(0)$
\end{tabular}

\section{Disease control and survival}

The median follow-up for all the patients after completing the reirradiation was 17 months (range, 2-59 months). At the time of the last follow-up, clinical local control had been achieved in 7 patients (31.8\%), and 13 patients had developed distant metastases. The cumulative local progression-free survival rates were $67.0 \%(\mathrm{SE}, \pm 10.3 \%)$ at 1 year post-reirradiation and $10.7 \%(\mathrm{SE}, \pm 9.2 \%)$ at 2 years post-reirradiation. The median local control time was 14 months. Eighteen patients (81.8\%) died, and the cumulative overall survival rates were $85.9 \%(\mathrm{SE}, \pm 7.6 \%)$ at 1 year post-reirradiation and $27.2 \%$ (SE, $\pm 10.3 \%$ ) at 2 years post-reirradiation. The median survival time after reirradiation was 19 months.

\section{Discussion}

The published literature offers limited data on the use of reirradiation for treating recurrent rectal cancer in patients with previous pelvic irradiation. To the best of our knowledge, no previous prospective studies have evaluated the efficacy and safety of accelerated hyperfractionated IMRT in recurrent rectal cancer patients with previous pelvic irradiation. This study determined whether accelerated hyperfractionated IMRT is effective and safe for treating recurrent/unresectable rectal cancer in patients with previous pelvic irradiation. The overall local response rate was $40.9 \%$, and the clinical symptom relief rate was $86.4 \%$. Grade 4 acute toxicity and treatment-related deaths were not observed. Grade 3 diarrhea, cystitis, radiation dermatitis, and intestinal obstruction were observed in $9.1 \%$, $4.5 \%, 4.5 \%$, and $4.5 \%$ of the participants, respectively. Late toxicity was infrequent and mild.

Reirradiation for recurrent rectal cancer has historically been unacceptable for patients with previous pelvic irradiation because of the extremely high incidence of complications in normal tissues. Data on reirradiation are scarce. However, several recent reports and trials have demonstrated the safety and efficacy of reirradiation for treating recurrent rectal cancer in patients with previous pelvic irradiation [8,10-13]. Although a high reirradiation dose cannot be applied to previously irradiated regions, reirradiation doses of 30-40 Gy are well tolerated and provide decent palliation in most patients. Recently, hyperfractionated reirradiation has been used for recurrent rectal cancer to reduce the risk for late toxicity in normal tissues related to previous irradiation. Several studies have suggested that hyperfractionated reirradiation is a valuable treatment option that provides effective palliation with an acceptable toxicity profile $[11,12]$. Additionally, to reduce toxicity in normal tissues, IMRT has been recommended when reirradiating patients with recurrent rectal cancer [14]. Several dosimetric and clinical studies have shown that IMRT for rectal cancer can reduce the dose delivered to the small bowel (thereby reducing treatment-related toxicity) compared with standard 3DCRT $[16,17]$. Based on these considerations, accelerated hyperfractionated IMRT was used to treat patients with recurrent rectal cancer after previous pelvic irradiation.

Although reirradiation concurrent with chemotherapy may lead to better clinical outcomes, we did not evaluate this regimen in our study. Mohiuddin et al. [10] reported high toxicity in patients concurrently treated with reirradiation and continuous 5-FU infusion. In their study, $28 \%$ of the patients developed grade 3 to 4 acute toxicity during reirradiation, and $21 \%$ of the patients developed late complications, such as chronic severe diarrhea and small bowel obstruction. This acute toxicity required interrupting or terminating the treatment in $22 \%$ of the patients. In our study, the total reirradiation course was only 3 weeks, and the impact of concurrent chemotherapy may have been small. Moreover, in our study, the intent of reirradiating patients with inoperable recurrent rectal cancer was palliative.

Our study achieved an overall local response rate of $40.9 \%$. None of the patients achieved a CR, mainly due to the large tumor sizes $(77.3 \%$ of the patients had lesions larger than $3 \mathrm{~cm}$ ) and the low total reirradiation dose. These findings are comparable to those reported in previous trials. Valentini et al. [11] reported results from a multicenter phase II study of reirradiation in 59 patients with recurrent rectal cancer. These patients were treated twice daily with 1.2-Gy fractions for a cumulative dose of $40.8 \mathrm{~Gy}$ and with concurrent continuous 5 -FU infusion. The authors reported a $44.1 \%$ overall response rate (CR, 8.5\%; PR, 35.6\%). Another retrospective study [13] investigated reirradiation in 22 patients with recurrent rectal cancer who had previously received pelvic irradiation. The overall response rates at 1 and 3 months were $32 \%$ and $41 \%$, respectively. A CR was observed in one patient (5\%) at 3 months. The percentage of PRs increased from $27 \%$ at 1 month to $37 \%$ at 3 months.

Reirradiation provided effective symptomatic palliation, even at the lower total dose that was administered to the recurrent rectal tumors. In our study, the clinical symptom relief rate was $86.4 \%$ (complete symptom relief rate, $27.3 \%$; partial symptom relief rate, $59.1 \%$ ). The proportion of patients achieving clinical symptomatic relief 
was similar to that reported in several other reirradiation studies. Mohiuddin et al. [10] reported that bleeding, pain, and the effects of the tumor were positively impacted in $100 \%, 82.6 \%$, and $88.9 \%$ of the patients, respectively, after reirradiation. Valentini et al. [11] also reported a high symptom relief rate (83.3\%) 4 weeks after the end of the reirradiation and concurrent chemotherapy.

In our study, the toxicity data revealed that accelerated hyperfractionated IMRT was well tolerated, with low rates of acute and late toxicity, in patients with recurrent rectal cancer and previous pelvic irradiation. Diarrhea was the most common acute toxicity; late toxicity was infrequent and mild. Various acute and late toxicities have been described in the literature, and most studies have reported a low incidence of toxicity, similar to our results [11,13]. However, Mohiuddin et al. [10] reported that $22 \%$ of the patients required a significant treatment interruption, and $15 \%$ of the patients terminated treatment due to severe acute toxicities. Additionally, grade 4 diarrhea was observed in $6 \%$ of the patients, and late complications were reported for $21.4 \%$ of the patients. Das et al. [12] also reported a high incidence of grade 3 to 4 late toxicity (26\%). The incidence of reirradiation toxicity is correlated with many factors, such as reirradiation dose, reirradiation fractionation, total cumulative dose, the amount of small bowel that is exposed to the radiation field, reirradiation intervals, and the previous radiation and reirradiation volumes. When reirradiating the pelvis to treat recurrent rectal cancer, every effort should be made to limit the dose to the small bowel and bladder in the radiation field.

Although reirradiation of recurrent rectal cancer can result in excellent palliation of symptoms and good local responses with acceptable toxicity, the long-term local control and survival remain poor. To improve these factors, other approaches, such as radiotherapy dose escalation, radiotherapy combined with newer chemotherapeutic and biological agents, intraoperative radiotherapy, hyperthermia, radical surgery, and systemic treatment, should be investigated in future studies.

Our study had several limitations. One limitation is that the sample size was small. Furthermore, late toxicity may have been incompletely assessed based on medical records. Moreover, some patients who might have developed late toxicity died prematurely. Therefore, the late toxicity rates may have been underestimated.

In conclusion, accelerated hyperfractionated IMRT can provide excellent symptom palliation and a good local response in patients with recurrent/unresectable rectal cancer and previous pelvic irradiation. The toxicity profiles for the dosage and schedule used in our study were acceptable. Additional studies are necessary to develop innovative treatment regimens and improve the clinical outcome while avoiding excessive toxicity in patients with recurrent rectal cancer who received previous pelvic irradiation.

\section{Competing interests}

The authors declare that they have no competing interests.

\section{Authors' contributions}

GC, JZ and ZZ carried out study design, participated in collection and assembly of data, data analysis, manuscript writing. WGH carried out radiation treatment planning. All authors read and approved the final manuscript.

Received: 18 July 2014 Accepted: 26 November 2014 Published online: 11 December 2014

\section{References}

1. van Gijn W, Marijnen CA, Nagtegaal ID, Kranenbarg EM, Putter $H$, Wiggers T, Rutten HJ, Pahlman L, Glimelius B, van de Velde CJ, Dutch Colorectal Cancer G: Preoperative radiotherapy combined with total mesorectal excision for resectable rectal cancer: 12-year follow-up of the multicentre, randomised controlled TME trial. Lancet Oncol 2011, 12:575-582.

2. How P, Shihab O, Tekkis P, Brown G, Quirke P, Heald R, Moran B: A systematic review of cancer related patient outcomes after anterior resection and abdominoperineal excision for rectal cancer in the total mesorectal excision era. Surg Oncol 2011, 20:e149-155.

3. Bouchard P, Efron J: Management of recurrent rectal cancer. Ann Surg Oncol 2010, 17:1343-1356.

4. Sebag-Montefiore D, Stephens RJ, Steele R, Monson J, Grieve R, Khanna S, Quirke P, Couture J, de Metz C, Myint AS, Bessell E, Griffiths G, Thompson LC, Parmar M: Preoperative radiotherapy versus selective postoperative chemoradiotherapy in patients with rectal cancer (MRC CR07 and NCIC-CTG C016): a multicentre, randomised trial. Lancet 2009, 373:811-820.

5. Garcia-Aguilar J, Cromwell JW, Marra C, Lee SH, Madoff RD, Rothenberger DA: Treatment of locally recurrent rectal cancer. Dis Colon Rectum 2001, 44:1743-1748.

6. Camilleri-Brennan J, Steele RJ: The impact of recurrent rectal cancer on quality of life. Eur J Surg Oncol 2001, 27:349-353.

7. Nielsen MB, Laurberg S, Holm T: Current management of locally recurrent rectal cancer. Colorectal Dis 2011, 13:732-742.

8. Lingareddy V, Ahmad NR, Mohiuddin M: Palliative reirradiation for recurrent rectal cancer. Int J Radiat Oncol Biol Phys 1997, 38:785-790.

9. Mohiuddin M, Marks GM, Lingareddy V, Marks J: Curative surgical resection following reirradiation for recurrent rectal cancer. Int J Radiat Oncol Biol Phys 1997, 39:643-649.

10. Mohiuddin M, Marks G, Marks J: Long-term results of reirradiation for patients with recurrent rectal carcinoma. Cancer 2002, 95:1144-1150.

11. Valentini V, Morganti AG, Gambacorta MA, Mohiuddin M, Doglietto GB, Coco C, De Paoli A, Rossi C, Di Russo A, Valvo F, Bolzicco G, Dalla Palma M: Preoperative hyperfractionated chemoradiation for locally recurrent rectal cancer in patients previously irradiated to the pelvis: a multicentric phase II study. Int J Radiat Oncol Biol Phys 2006, 64:1129-1139.

12. Das P, Delclos ME, Skibber JM, Rodriguez-Bigas MA, Feig BW, Chang GJ, Eng C, Bedi M, Krishnan S, Crane CH: Hyperfractionated accelerated radiotherapy for rectal cancer in patients with prior pelvic irradiation. Int J Radiat Oncol Biol Phys 2010, 77:60-65.

13. Koom WS, Choi Y, Shim SJ, Cha J, Seong J, Kim NK, Nam KC, Keum KC: Reirradiation to the pelvis for recurrent rectal cancer. J Surg Oncol 2012, 105:637-642.

14. Benson AB 3rd, Bekaii-Saab T, Chan E, Chen YJ, Choti MA, Cooper HS, Engstrom PF, Enzinger PC, Fakih MG, Fuchs CS, Grem JL, Hunt S, Leong LA, Lin E, Martin MG, May KS, Mulcahy MF, Murphy K, Rohren E, Ryan DP, Saltz L, Sharma S, Shibata D, Skibber JM, Small W Jr, Sofocleous CT, Venook AP, Willett CG, Freedman-Cass DA, Gregory KM: Rectal cancer. J Natl Compr Canc Netw 2012, 10:1528-1564.

15. Hodapp N: The ICRU Report 83: prescribing, recording and reporting photon-beam intensity-modulated radiation therapy (IMRT). Strahlenther Onkol 2012, 188:97-99.

16. Guerrero Urbano MT, Henrys AJ, Adams EJ, Norman AR, Bedford JL, Harrington KJ, Nutting CM, Dearnaley DP, Tait DM: Intensity-modulated 
radiotherapy in patients with locally advanced rectal cancer reduces volume of bowel treated to high dose levels. Int I Radiat Oncol Biol Phys 2006, 65:907-916.

17. Samuelian JM, Callister MD, Ashman JB, Young-Fadok TM, Borad MJ, Gunderson LL: Reduced acute bowel toxicity in patients treated with intensity-modulated radiotherapy for rectal cancer. Int J Radiat Oncol Biol Phys 2012, 82:1981-1987.

doi:10.1186/s13014-014-0278-3

Cite this article as: Cai et al:: Accelerated hyperfractionated intensity-

modulated radiotherapy for recurrent/unresectable rectal cancer in patients

with previous pelvic irradiation: results of a phase II study. Radiation Oncology 2014 9:278.

\section{Submit your next manuscript to BioMed Central and take full advantage of:}

- Convenient online submission

- Thorough peer review

- No space constraints or color figure charges

- Immediate publication on acceptance

- Inclusion in PubMed, CAS, Scopus and Google Scholar

- Research which is freely available for redistribution 